FCl24 Psychopathology and psychotherapies

\section{Clinical features of Lewy Body Dementia}

Luigı Ravizza, Alessandra Bada, Cristına Bertolı, Filıpo Bogetto, Giuseppe Maına

Dept. of Neuroscience, Psychistric Unit, University of Turin

Lewy Body Dementıa is now considered a quite common cause of dementua of uncertain nosological status. According to several authors, it represents a neuropathologically distinct subset of Alzheimer's disease (AD); for other authors, Diffuse Lewy Body Desease (DLB) is a distinct type of desease that should be considered as an autonomous disorder

The aim of the present sudy was to investigate on the clinıcal features of a group of patients with Senile Dementia of Alzheimer Type (SDAT) in order to identify all subjects with probable Lewy Body Dementia.

All subjects were at least 65 years old and diagnosed with SDAT according to DSM-III-R. The patients were assessed at their own place using a semistructured interview, the Gottfriés-Brane-Steen Rating Scale for Dementia (GBS), the Hamilton Depression Rating Scale, the Minı Mental State, the Clock Drawing Test. A neurological examination to evaluate extrapyramidal signs was also performed in all patients.

12 clinically diagnosed SDAT patients were considered as probable DLB. The patients with Lewy Body Dementia appeared to constutute a distinct clinical entity: They did not differ significantly regarding age, gender or education, but several differences in symptomatology and in the illness course were found. The DLB subjects exhibited a greater degree of visuospatial/constructional impairment that did the $A D$ subjects with a higher degree of fluctuation during the day of the cognitive impaiment. In addition, DLB was associated with a more severe clinical course.

Our findings will be discussed together with the other data that have recently appeared in literature.
FC126 Psychopathology and psychotherapies THE DYNAMICS OF PLENARY GROUPS IN THE HOSPITAL FOR DYNAMIC PSYCHIATRY

\section{R. Schmidts. Dynamic Psychiatric Hospital Menterschwaige,} Geiselgasteigstrasse 203, Munich, Germany.

On the basis of human structural multidimensionality the plenary group develops a network of relationships and a therapeutic field which integrates the participants of the group. The interpersonal field of the plenary group created by the group members in an obvious way integrates an energetic processual development by teaching the individual to realize hisher unique identity and hence hisher importance for the group. The plenary group is a group dynamic figure in which a most intensive exchange of information takes place on general events of the hospital and on peculiarities and concerns of individuals. This is why it is an extraordinary field for meeting each other for experience of time, and for the right way of thinking which develops within relationships. The plenary group suppons and confurms the identity of a person and the identity of formal and informal small groups. It develops norms of group boundaries, which again and again enact and develop above all the values of their therapeutic culture. Dialectically bound to its legal character it is also a group which accounts for the human need to have festivals. Its therapeutic effect for inpatient psychotherapy is therefore a fundamental one within the many dimensions of human possibilities.

\section{$\mathrm{FCl} 25$ Psychopathology and psychotherapies THE ANALYSIS OF THERAPEUTIC EFFECTS OF ALCOHOL DEPENDENCY IN PATIENT TREATMENT}

M. Rus-Makovec. Department of Alcohol Dependency Treatment, Mental Health Center, Poljanski Nasip 58, Luubljana, Slovenia.

The aim of the study was to differentiate and evaluate the effects of in-patient group treatment of alcohol dependent patients on variables, relevant for successful outcome. 169 patients participated in the study. The research plan was a modified version of Solomon's experimental plan. In group $1(n=71)$ there were patients tested only in the end of the treatment, in group $2(n=69)$ in the beginning and at the end of the treatment and in group $3(n=29)$ there were patients who relapsed (tested only in the beginning). Relating to chosen theoretical model of successful oulcome, dependene variables were self-evaluation, the perception of saturation of one's own needs, the perception of norms, the perception of harmfulness of alcohol, expectations from treatment, behavioral intentions about sober behavior. The perception of group climate and of leadership style were independent variables. In the course of therapy the patients acquired a more positive attitude towards themselves and they displayed an upward trend in active, self-responsible behavior. A more positive perception of the group climate was related to more favorable treatment indices. The results made it possible to establish the subgroups of more vulnerable patients and to formulate important therapeutic directions.

\section{FC127 Psychopathology and psychotherapies PATIENT'S SUBJECTIVE ESTIMATION OF FACTORS THAT INFLUENCE THEIR WELL-BEING}

M. Sitarz, A. Popiel. II Department of Psychiatry, Warsaw Medical School, Nowowiejska 27, Warsaw, Poland.

The aim of the work is the analysis of the factors which are subjectively described by the patients as those which influenced the process of change of their delusional statements. The authors have examined a group of patients with DSM-IV diagnosis of schizophrenia who were assessed as "much improved" or "very much improved" according to the Clinical Global Impression Scale by the use of the semi-structured interview. The factors which were listed by the patients were then divided into several groups: environmental (Nursing staff, doctors, psychologists, family, other patients): pharmacotherapeutic; patient's personal experience (his observations, lectures, thoughts, the fact of hospitalisation); others. They were then analysed in the context of delusions and subjective assessment of the patients' well-being. 